\title{
Erratum: \\ Symmetric Iterative Interpolation Processes
}

\author{
Gilles Deslauriers and Serge Dubuc
}

As Professor Charles A. Micchelli has kindly pointed out to us, the proof of Theorem 6.4 in [1] is incorrect. We state again this theorem and we supply a satisfactory proof. We use the same notation, all references in the proof use previous results of the same article.

Theorem 6.4. The fundamental function $F(t)$ of an interpolation process of type $(b, N)$ has a unique continuous extension to the real axis. The Fourier transform of that extension is $G(\xi)$.

Proof. Since $G(\xi)$ is integrable (see Theorem 6.3), we define the continuous function $H(x)=(1 / 2 \pi) \int G(\xi) e^{i x \xi} d \xi$. The basic step of the proof is to show that $H(m)=F(m)$ for any integer $m$. Let us consider the following sequence of functions: $f_{0}(\xi) \equiv 1$ and, for $n>0, f_{n}(\xi)=\prod_{k=1}^{m}\left[P\left(-\xi / b^{k}\right) / b\right]$ if $|\xi| \leq \pi b^{n}$, otherwise $f_{n}(\xi)=0$. From the fact that $G(\xi)=\prod_{k=1}^{\infty}\left[P\left(-\xi / b^{k}\right) / b\right]$, an absolutely convergent infinite product, the zeros of $G$ are those of its factors $\left[P\left(-\xi / b^{k}\right) / b\right]$; it follows that $G(\xi)$ is positive on $[-\pi, \pi]$. If the number $A$ is chosen as

$$
\sup \left\{f_{n}(\xi): \xi \in[-\pi, \pi], n=0,1,2, \ldots\right\} / \min \{G(\xi): \xi \in[-\pi, \pi]\},
$$

then $f_{n}(\xi) \leq A G(\xi)$, for $n=0,1,2, \ldots$, and for any $\xi$ in $[-\pi, \pi]$. If $\xi$ is a number in $\left[-\pi b^{m}, \pi b^{m}\right]$ and if $n \geq m$, then

$$
f_{n}(\xi)=\prod_{k=1}^{m}\left[P\left(-\xi / b^{k}\right) / b\right] f_{n-m}\left(\xi / b^{m}\right) \leq \prod_{k=1}^{m}\left[P\left(-\xi / b^{k}\right) / b\right] A G\left(\xi / b^{m}\right)=A G(\xi) .
$$

The last equality is a consequence of the functional equation (5.2). The sequence $\left\{f_{n}\right\}$ is dominated by the integrable function $A G$. From the Lebesgue dominated convergence theorem, $H(m)$ is the limit of $(1 / 2 \pi) \int_{-\pi b^{n}}^{\pi b^{n}} f_{n}(\xi) e^{i m \xi} d \xi$ when $n$ tends to

Date received: December 3, 1990. Date revised: June 5, 1991. Communicated by Charles A. Micchelli. 
$\infty$. According to formula $(5.1), \prod_{k=0}^{n-1} P\left(b^{k} \theta\right)=\sum_{k} F\left(k / b^{n}\right) e^{i k \theta}$, so

$$
\frac{1}{2 \pi} \int_{-\pi b^{n}}^{\pi b^{n}} f_{n}(\xi) e^{i m \xi} d \xi=F(m) \text { and } H(m)=F(m) .
$$

From the functional relation $(5.2), G(b \xi)=G(\zeta) P(-\xi) / b$, it follows that $H(x / b)=$ $\sum_{k} F(k / b) H(x-k)$. By induction, $H(x)=F(x)$ for any $b$-adic number. $H$ is the continuous extension of $F$ and $H$ has compact support, it is integrable, and its Fourier transform cannot be different from $G$.

\section{Reference}

1. G. Deslauriers, S. Dubuc (1989): Symmetric iterative interpolation processes. Constr. Approx. 5:49-68. 